\title{
Association of the location of pancreatic ductal adenocarcinoma (head, body, tail) with tumor stage, treatment, and survival: a population-based analysis
}

Felice N. van Erning, Tara M. Mackay, Lydia G. M. van der Geest, B. Groot Koerkamp, Hanneke W. M. van Laarhoven, Bert A. Bonsing, Johanna W. Wilmink, Hjalmar C. van Santvoort, Judith de Vos-Geelen, Casper H. J. van Eijck, Olivier R. Busch, Valery E. Lemmens, Marc G. Besselink \& for the Dutch Pancreatic Cancer Group

To cite this article: Felice N. van Erning, Tara M. Mackay, Lydia G. M. van der Geest, B. Groot Koerkamp, Hanneke W. M. van Laarhoven, Bert A. Bonsing, Johanna W. Wilmink, Hjalmar C. van Santvoort, Judith de Vos-Geelen, Casper H. J. van Eijck, Olivier R. Busch, Valery E. Lemmens, Marc G. Besselink \& for the Dutch Pancreatic Cancer Group (2018): Association of the location of pancreatic ductal adenocarcinoma (head, body, tail) with tumor stage, treatment, and survival: a population-based analysis, Acta Oncologica, DOI: 10.1080/0284186X.2018.1518593

To link to this article: https://doi.org/10.1080/0284186X.2018.1518593

a

(C) 2018 The Author(s). Published by Informa UK Limited, trading as Taylor \& Francis Group.

曲 Published online: 28 Sep 2018.

Llll Article views: 128

(

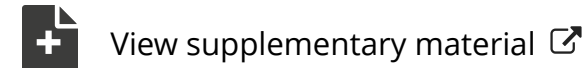

View supplementary material $[7$

Submit your article to this journal 


\title{
Association of the location of pancreatic ductal adenocarcinoma (head, body, tail) with tumor stage, treatment, and survival: a population-based analysis
}

\author{
Felice N. van Erning ${ }^{\mathrm{a} *}$, Tara M. Mackay ${ }^{\mathrm{b} *}$ (D) Lydia G. M. van der Geest ${ }^{\mathrm{a}}$, B. Groot Koerkamp ${ }^{\mathrm{c}}$, \\ Hanneke W. M. van Laarhoven ${ }^{d}$, Bert A. Bonsing ${ }^{e}$, Johanna W. Wilmink ${ }^{d}$, Hjalmar C. van Santvoort ${ }^{f}$, \\ Judith de Vos-Geelen ${ }^{\mathrm{g}}$, Casper H. J. van Eijck ${ }^{\mathrm{c}}$, Olivier R. Busch ${ }^{\mathrm{b}}$, Valery E. Lemmens ${ }^{\mathrm{a}, \mathrm{h} * *}$, Marc G. Besselink ${ }^{\mathrm{b} * *}$ \\ and for the Dutch Pancreatic Cancer Group
}

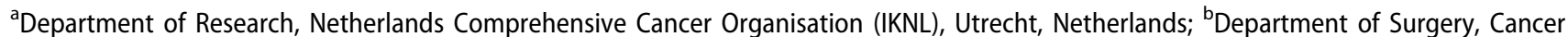
Center Amsterdam, Amsterdam UMC, University of Amsterdam, Amsterdam, Netherlands; 'Department of Surgery, Erasmus Medical Center, Rotterdam, the Netherlands; ${ }^{\mathrm{d} D e p a r t m e n t}$ of Medical Oncology, Cancer Center Amsterdam, Amsterdam UMC, University of Amsterdam, Amsterdam, the Netherlands; ${ }^{\text {}}$ Department of Surgery, Leiden University Medical Center, Leiden, the Netherlands; ${ }^{\mathrm{f} D e p a r t m e n t}$ of Surgery, Regional Academic Cancer Center Utrecht, University Medical Center Utrecht Cancer Center \& St. Antonius Hospital Nieuwegein, Nieuwegein, the Netherlands; ${ }^{9}$ Department of Internal Medicine, Division of Medical Oncology, GROW - School for Oncology and

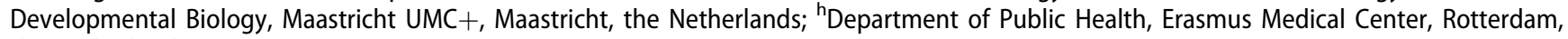
the Netherlands
\end{abstract}

\begin{abstract}
Background: The association between pancreatic ductal adenocarcinoma (PDAC) location (head, body, tail) and tumor stage, treatment and overall survival (OS) is unclear.

Methods: Patients with PDAC diagnosed between 2005 and 2015 were included from the populationbased Netherlands Cancer Registry. Patient, tumor and treatment characteristics were compared with the tumor locations. Multivariable logistic and Cox regression analyses were used.

Results: Overall, 19,023 patients were included. PDAC locations were 13,451 (71\%) head, 2429 (13\%) body and $3143(16 \%)$ tail. Differences were found regarding metastasized disease (head $42 \%$, body $69 \%$, tail $84 \%, p<.001)$, size $(>4 \mathrm{~cm}: 21 \%, 40 \%, 51 \%, p<.001)$ and resection rate $(17 \%, 4 \%, 7 \%$, $p<.001$ ). For patients without metastases, median OS did not differ between head, body, tail (after resection: 16.8, 15.0, 17.3 months, without resection: 5.2, 6.1, 4.6 months, respectively). For patients with metastases, median OS differed slightly $(2.6,2.4,1.9$ months, respectively, adjusted HR body versus head 1.17 (95\%Cl 1.10-1.23), tail versus head 1.35 (95\% Cl 1.29-1.41)).

Conclusions: PDAC locations in body and tail are larger, more often metastasized and less often resectable than in the pancreatic head. Whereas survival is similar after resection, survival in metastasized disease is somewhat less for PDAC in the pancreatic body and tail.
\end{abstract}

ARTICLE HISTORY

Received 9 May 2018

Accepted 23 August 2018

\section{Background}

Patients with pancreatic ductal adenocarcinoma (PDAC) have a median overall survival of only 4-6 months [1] and a 5year overall survival of $7 \%$ [2]. Only $20 \%$ of the patients are amenable to resection. Following resection, the median overall survival is 17-21 months and the 5-year overall survival is 16-19\% [3-5]. When resection can be combined with adjuvant chemotherapy, median overall survival increases from 7 months to 26-28 months [6]. Survival with PDAC is associated with different factors, such as tumor biology, stage and possible treatment. One of the issues that is still debated is the association of the primary tumor location with survival.
Data from the Surveillance, Epidemiology and End Results (SEER) database showed that patients with PDAC in the pancreatic head have higher 3-year relative survival rates than patients with a PDAC in the pancreatic body or tail in case of regional $(7.6 \%$ versus $6.7 \%$ ) or distant disease $(1.8 \%$ versus $1.4 \%)$. For local disease, results were reversed $(9.0 \%$ versus $20 \%$ [7]. Another US study investigating the disease-specific survival by tumor location, reported higher survival rates for PDAC in the tail than for PDAC in the head for all the stages, while survival for PDAC in the body was higher than for PDAC in the head in stage I, but lower in stages II-IV [8]. A study including patients from a single institution in the US who underwent resection for PDAC in the period of

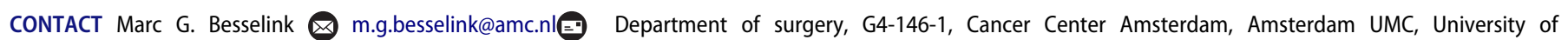
Amsterdam, Meibergdreef 9, PO Box 22660, 1105 AZ Amsterdam, Netherlands

*Shared first author; these authors contributed equally to this work

**Shared senior author; these authors contributed equally to this work

(1) Supplemental data for this article can be accessed here.

(C) 2018 The Author(s). Published by Informa UK Limited, trading as Taylor \& Francis Group.

This is an Open Access article distributed under the terms of the Creative Commons Attribution-NonCommercial-NoDerivatives License (http://creativecommons.org/licenses/by-nc-nd/4. $0 /$, which permits non-commercial re-use, distribution, and reproduction in any medium, provided the original work is properly cited, and is not altered, transformed, or built upon in any way. 
1994-2014, reported similar oncological outcomes between the tumor locations [5]. However, two of the three studies did not have recent study periods (1973-2002 and 1985-1995).

Due to the lack of early symptoms due to biliary obstruction, PDAC in the body or tail are usually more advanced and are larger at diagnosis than PDAC in the head [5,9]. This may be the sole explanation for a worse survival in PDAC in the body or tail. However, a recent clinicopathological study from Glasgow reported that PDAC of the body and tail may have a more aggressive tumor biology compared to PDAC of the head. PDAC of the body and tail was associated with the PDAC squamous subtype and enriched for gene programmes associated with inflammation, epithelial-to-mesenchymal transition and potential immune avoidance mechanisms, which may also explain worse outcomes in these patients [10].

The aim of this study is, therefore, to investigate tumor, patient and treatment characteristics and outcomes of PDAC in the head, body and tail of all stages in the Netherlands.

\section{Methods}

\section{Data collection}

This is a retrospective study using data from the nationwide Netherlands Cancer Registry. The Netherlands Cancer Registry is a population-based registry which collects data on all newly diagnosed cancer patients in the Netherlands. Information on patient and tumor characteristics, diagnosis and treatment is routinely extracted from the medical records by trained administrators of the Netherlands Cancer Registry. Anatomical site of the tumor is registered according to the International Classification of Disease - Oncology (ICDO). The TNM (tumor-node-metastasis) classification is used for stage notification of the primary tumor, according to the edition valid at the time of cancer diagnosis. In case of resection, margin status was classified as microscopically radical resection ( $>1 \mathrm{~mm}$; $\mathrm{R0}$ ), microscopically irradical resection ( $\leq 1 \mathrm{~mm} ; \mathrm{R} 1)$, and macroscopic irradical resection (R2). Survival data is obtained by annual linkage with the Municipal Personal Records Database, which contains information on the vital status of all Dutch inhabitants, and is completed until 1 February 2017.

\section{Study population}

For the present study, all the patients with pancreatic ductal adenocarcinoma (PDAC) or suspected PDAC (ICD-O C25, morphology codes 8010, 8012, 8020, 8140, 8141, 8260, 8310, $8440,8480,8481,8490,8500$, and 8560 , or a non-microscopic verified invasive neoplasm of the pancreas suspected for adenocarcinoma (i.e., no cytological or histological verification) diagnosed between 2005 and 2015 were selected. Patients younger than 18 years at diagnosis or residing abroad and patients diagnosed at autopsy were excluded. Tumor stage was based on pathological TNM stage (for patients who underwent resection) supplemented by clinical
TNM stage if no pathological TNM stage was available (for patients without resection; 2005-2009 TNM-6, 2010-2015 TNM-7). Until 2011, a summary stage was recorded for nonverified cancers. Therefore, tumor stage of all patients was categorized as: locoregional (tumor confined to the pancreas or extended to surrounding organs, tissues or regional lymph nodes), distant metastases (tumor spread to distant lymph nodes or organs) and unknown. Patients with distant metastases who underwent resection were excluded. Patients were also classified according to tumor location: head (C25.0), body (C25.1), tail (C25.2) and other (C25.3-9). The latter group was excluded. For treatment and survival analysis, patients who underwent resection, patients without resection and without distant metastases, and patients with distant metastases were analyzed separately.

\section{Treatment and outcome measures}

Primary outcome of the study was overall survival by tumor location in relation to resection. For patients undergoing resection, secondary outcomes included histopathological margin status, $\geq 10$ examined lymph nodes, number of positive lymph nodes, and 30- and 90-day mortality. Type of resection and the proportions of preoperative and adjuvant chemotherapy were also investigated. For patients without resection, the proportion of patients undergoing chemotherapy was defined.

\section{Statistical analyses}

Descriptive statistics were used to compare patient, tumor and treatment characteristics for all patients and for patients who underwent resection. Characteristics included gender, age, tumor stage, microscopic verification and tumor size (the latter only available in 2015). Included treatments were resection (mainly pancreatoduodenectomy and distal pancreatectomy), preoperative, adjuvant and palliative chemotherapy. Multivariable logistic regression analyses, presented as odds ratios with $95 \%$ confidence intervals, were used to determine the independent association of tumor location with the likelihood of receiving resection (in all patients) and chemotherapy (in patients who underwent resection, patients without resection and without distant metastases, and patients with distant metastases separately). Differences in 2-year overall survival according to tumor location were analyzed by means of Kaplan-Meier curves and compared with Log-Rank tests. Multivariable Cox regression analyses were used to determine the independent association of tumor location with long-term survival and the results presented as hazard ratios with $95 \%$ confidence intervals. Survival time was defined as the time between the date of diagnosis (for patients without resection) or date of resection (for patients who underwent resection) and date of death or censoring (1 February 2017). The variables that were included in the multivariable analyses (as applicable) were gender, age (categorized as $<65,65-74$ and $\geq 75$ years), period of diagnosis (categorized as 2005-2008, 2009-2012 and 2013-2015), tumor stage, resection and preoperative, 


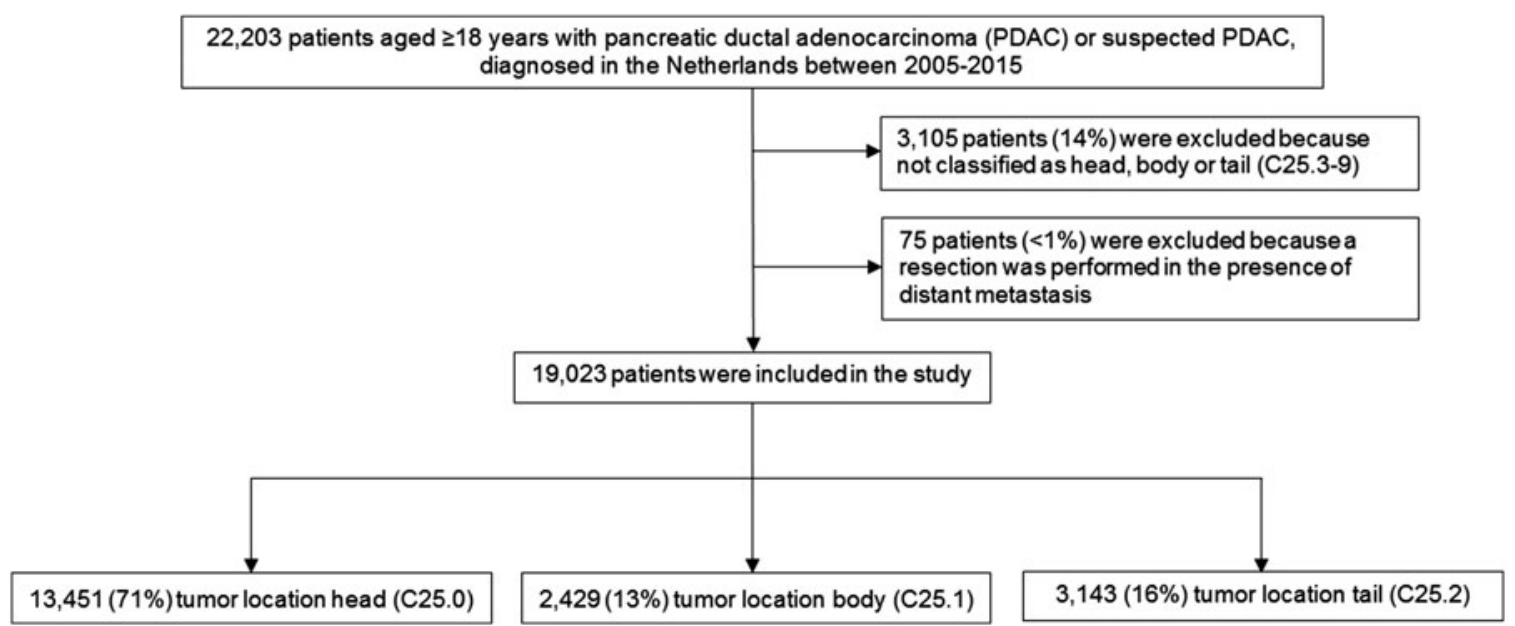

Figure 1. Selection of the study population.

adjuvant and palliative chemotherapy. To investigate whether the effect of tumor location on survival differed according to the stage, an interaction term was included in the model with all patients.

$p$ Values below .05 were considered to be statistically significant. SAS/STAT ${ }^{\circledR}$ statistical software (SAS system 9.4, SAS Institute, Cary, NY) was used for all the analyses.

\section{Results}

\section{Study population}

The final study population consisted of 19,023 patients with (suspected) PDAC: 13,451 patients $(71 \%)$ with PDAC in the pancreatic head, 2429 patients (13\%) in the body and 3143 patients (16\%) in the tail (Figure 1).

Table 1 provides an overview of the patient, tumor and treatment characteristics of the study population by tumor location. Large differences were especially found for tumor stage (i.e., proportion metastatic disease in head $42 \%$, body $69 \%$, tail $84 \%, p<.0001$ ), for tumor size (i.e., proportion tumor size $>4 \mathrm{~cm}$ in head $21 \%$, body $40 \%$, tail $51 \%$, $p<.0001$ ) and for microscopic verification (head $63 \%$, body $70 \%$, tail $75 \%, p<.0001)$.

For all the patients groups, chemotherapy rates were low (Table 2). Patients who received chemotherapy were younger than patients who did not receive chemotherapy, yet gender, tumor location and tumor size were similar in the three groups. Clinical characteristics of the patients with and without chemotherapy are provided in Supplementary Table S1. Overall, patients who received palliative chemotherapy had significantly better survival compared to patients without chemotherapy (median overall survival in patients without resection, no metastases with or without chemotherapy: 10.4 versus 4.3 months, and similarly, in patients without resection, but with metastases: 5.8 versus 1.6 months, respectively, both $p<.0001$, Supplementary Figure S1). Patients with a PDAC in the head more often underwent resection than patients with a PDAC in the body or tail $17 \%$ versus $4 \%$ versus $7 \%$, respectively, $p<.0001)$. After adjustment for gender, age, period of diagnosis, and tumor stage, patients with a
Table 1. Patient, tumor and treatment characteristics by PDAC location in the pancreas $(n=19,023)$.

\begin{tabular}{lcccc}
\hline & Head & Body & Tail & \\
& $n(\%)$ & $n(\%)$ & $n(\%)$ & $p$-value \\
\hline Total $n$ & 13451 & 2429 & 3143 & \\
Gender & & & & \\
$\quad$ Male & $6672(50)$ & $1158(48)$ & $1724(55)$ & $<.0001$ \\
$\quad$ Female & $6779(50)$ & $1271(52)$ & $1419(45)$ & \\
Age & & & & \\
$\quad<65$ years & $3909(29)$ & $795(33)$ & $1060(34)$ & $<.0001$ \\
$\quad 65-74$ years & $4231(31)$ & $831(34)$ & $1148(36)$ & \\
$\quad$ 75 years & $5311(40)$ & $803(33)$ & $935(30)$ & \\
Tumor stage & & & & \\
$\quad$ Locoregional & $7154(53)$ & $713(29)$ & $466(15)$ & $<.0001$ \\
$\quad$ Distant metastases & $5644(42)$ & $1671(69)$ & $2637(84)$ & \\
$\quad$ Unknown & $653(5)$ & $45(2)$ & $40(1)$ & \\
Microscopically verified & & & & \\
$\quad$ Yes & $8478(63)$ & $1706(70)$ & $2368(75)$ & $<.0001$ \\
$\quad$ No & $4973(37)$ & $723(30)$ & $775(25)$ & \\
Tumor size (2015 only) & & & & \\
$\quad$ 0-2 cm & $155(13)$ & $28(10)$ & $11(3)$ & $<.0001$ \\
$\quad 2-4$ cm & $618(51)$ & $98(34)$ & $97(27)$ & \\
$\quad>4$ cm & $249(21)$ & $118(40)$ & $182(51)$ & \\
$\quad$ Unknown & $188(15)$ & $48(16)$ & $67(19)$ & \\
Resection & & & & \\
$\quad$ Yes & $2320(17)$ & $107(4)$ & $207(7)$ & $<.0001$ \\
$\quad$ No & $11131(83)$ & $2322(96)$ & $2936(93)$ & \\
\hline
\end{tabular}

PDAC in the body were still less likely to undergo a resection, as compared to the patients with a PDAC in the head (adjusted odds ratio (OR) $0.29,95 \% \mathrm{Cl}$ 0.24-0.37), while patients with a PDAC in the tail were more likely to undergo a resection (adjusted OR tail versus head 1.46, 95\% Cl 1.19-1. 78) (Table 2).

For all stages combined, median overall survival was 4.5 months for PDAC in the head, 3.3 months for body and 2.2 months for tail (Figure 2(A)). The interaction test between tumor location and stage was significant $(p<.0001)$, indicating that the association between tumor location and overall survival differed according to stage. For tail versus head, survival was better in locoregional disease (HR $0.84,95 \% \mathrm{Cl}$ $0.76-0.93$ ) but worse in metastatic disease (HR $1.20,95 \% \mathrm{Cl}$ 1.15-1.26). For body versus head, survival was worse in locoregional disease (HR 1.15, 95\% Cl 1.06-1.24) but similar in metastatic disease (HR 1.02, 95\% Cl 0.97-1.08). 
Table 2. Logistic regression analyses predicting the provision of treatment.

\begin{tabular}{|c|c|c|c|c|}
\hline & Tumor location & Treated (\%) & Unadjusted OR $(95 \% \mathrm{Cl})$ & Adjusted OR $(95 \% \mathrm{Cl})$ \\
\hline \multicolumn{5}{|l|}{ All patients $(n=19023)$} \\
\hline \multirow[t]{3}{*}{ Treatment: resection } & Head & 17 & 1.00 (reference) & 1.00 (reference) $^{\mathrm{a}}$ \\
\hline & Body & 4 & $0.22(0.18-0.27)$ & $0.29(0.24-0.37)^{\mathrm{a}}$ \\
\hline & Tail & 7 & $0.34(0.29-0.39)$ & $1.46(1.19-1.78)^{\mathrm{a}}$ \\
\hline \multicolumn{5}{|c|}{ Patients who underwent resection $(n=2621)$} \\
\hline \multirow[t]{3}{*}{ Treatment: chemotherapy } & Head & 48 & 1.00 (reference) & 1.00 (reference) $^{b}$ \\
\hline & Body & 51 & $1.13(0.77-1.68)$ & $0.87(0.57-1.12)^{b}$ \\
\hline & Tail & 48 & $1.00(0.74-1.32)$ & $0.81(0.59-1.12)^{b}$ \\
\hline \multicolumn{5}{|c|}{ Patients without resection and without distant metastases $(n=5712)$} \\
\hline \multirow[t]{3}{*}{ Treatment: chemotherapy } & Head & 15 & 1.00 (reference) & 1.00 (reference) $^{\mathrm{b}}$ \\
\hline & Body & 30 & $2.47(2.04-2.99)$ & $2.03(1.64-2.51)^{b}$ \\
\hline & Tail & 20 & $1.42(1.03-1.94)$ & $1.24(0.88-1.74)^{b}$ \\
\hline \multicolumn{5}{|c|}{ Patients with distant metastases $(n=9952)$} \\
\hline \multirow[t]{3}{*}{ Treatment: chemotherapy } & Head & 22 & 1.00 (reference) & 1.00 (reference) $^{\mathrm{b}}$ \\
\hline & Body & 30 & $1.51(1.33-1.70)$ & $1.46(1.28-1.66)^{b}$ \\
\hline & Tail & 28 & $1.34(1.21-1.49)$ & $1.24(1.11-1.39)^{b}$ \\
\hline
\end{tabular}

Note: Bold text indicates statistically significant findings.

${ }^{a}$ Odds ratio adjusted for gender, age, period of diagnosis and tumor stage.

bodds ratio adjusted for gender, age, and period of diagnosis.

(A)

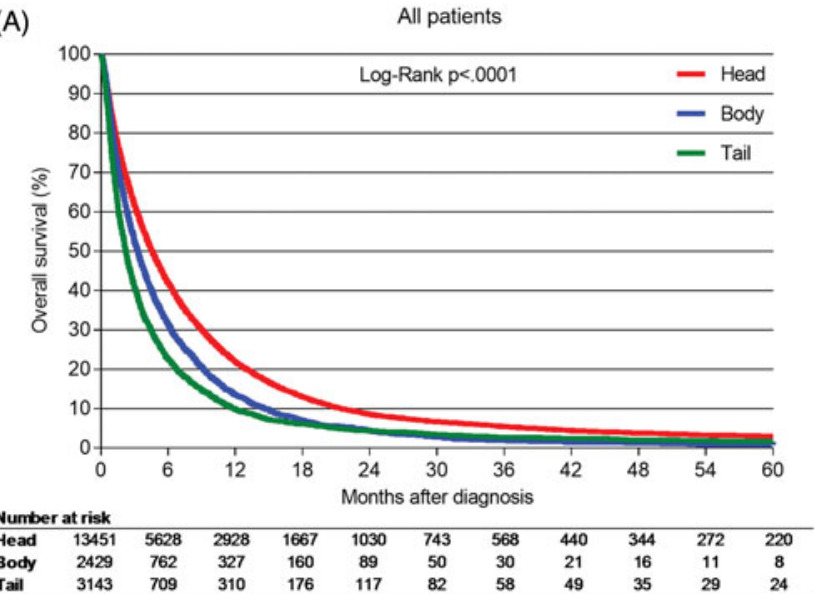

(C)

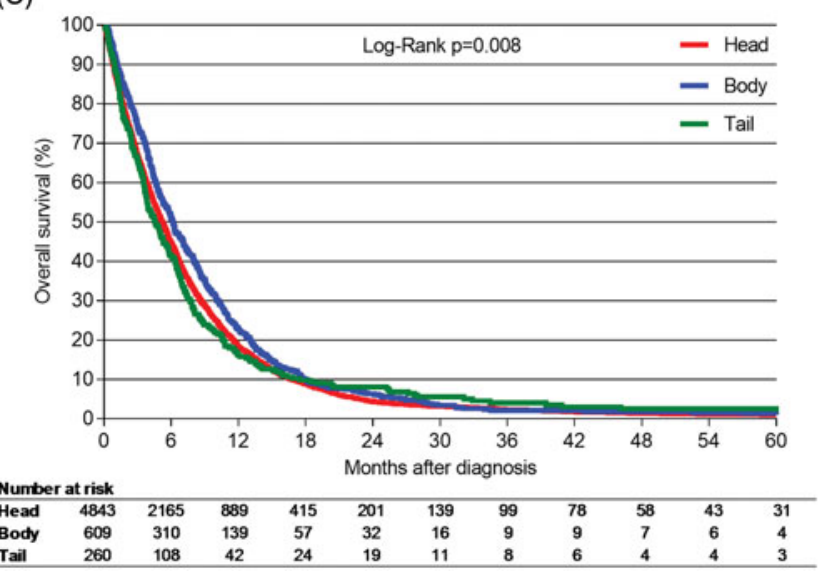

(B)

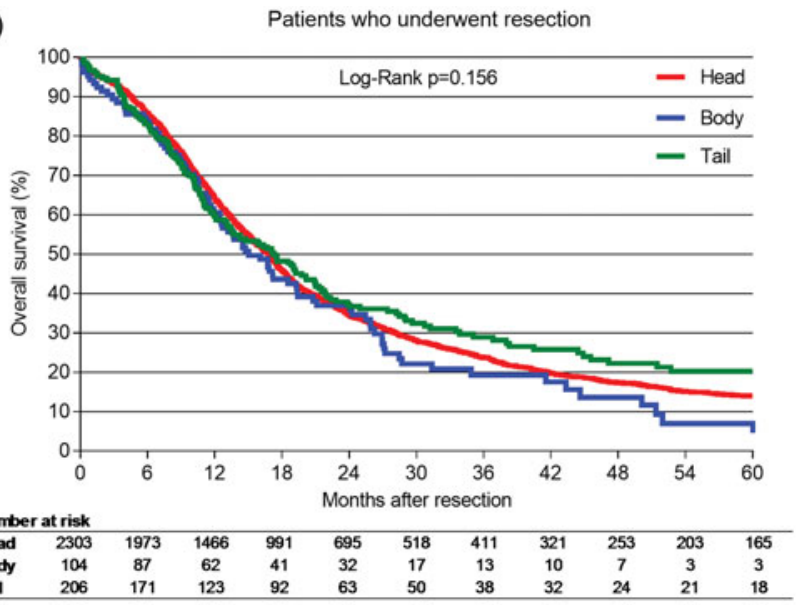

(D)

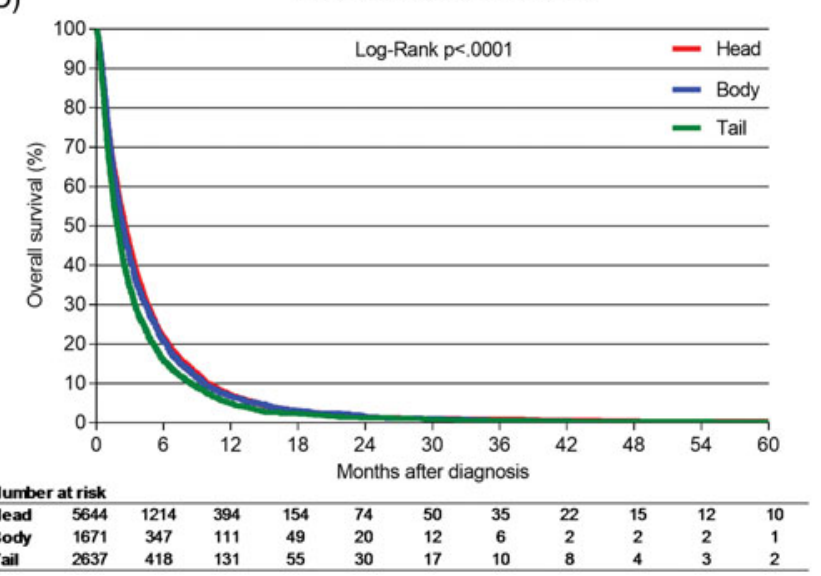

Figure 2. Overall survival by tumor location. Starting point survival analyses: A-C-D: date of diagnosis; B: date of resection.

\section{Patients who underwent resection}

Some 2621 patients underwent resection, additional characteristics of these patients per tumor location are presented in Table 3. In patients with a PDAC in the body, 32\% underwent a pancreatoduodenectomy and $61 \%$ a distal pancreatectomy. Margin status did not differ significantly between the tumor locations $(p=.066)$, as well as 30 or 90 day postoperative mortality ( $p=.501$ and $p=.256$ respectively). Approximately half of the patients received chemotherapy, regardless of tumor location (Table 2). Preoperative chemotherapy was mainly administered to patients with a PDAC in the body, while adjuvant chemotherapy was less often administered to the patients with a PDAC in the body (Table 3). In the group of patients who underwent resection, median overall survival was $16.8,15.0$ and 17.3 months, respectively for 
tumor location head, body and tail (Figure 2(B)). Two-year overall survival for head, body and tail were $36.2 \%, 36.9 \%$ and $37.9 \%$ respectively, while 5 -year overall survival was $14.1 \%$, $6.9 \%$ and $20.4 \%$ respectively. Also in multivariable analysis adjusting for patient, tumor and treatment characteristics, survival was similar for all tumor locations (Table 4).

\section{Patients without resection and without metastases}

The study population comprised 5712 patients without resection and without metastases. Of these patients, the

Table 3. Characteristics of patients undergoing resection of PDAC according to tumor location $(n=2,621 ; 2005-2015)$.

\begin{tabular}{|c|c|c|c|c|}
\hline & Head & Body & Tail & $p$-value* \\
\hline \multicolumn{5}{|l|}{ Pathological tumor stage } \\
\hline $\mathrm{T}_{1-3} N_{\text {any }}$ & $2176(94)$ & $98(94)$ & $194(94)$ & \multirow[t]{3}{*}{.858} \\
\hline $\mathrm{T}_{4} N_{\text {any }}$ & $103(5)$ & $5(5)$ & $11(5)$ & \\
\hline $\mathrm{T}_{\mathrm{X}} N_{\text {any }}$ & $32(1)$ & $1(1)$ & $1(1)$ & \\
\hline \multicolumn{5}{|l|}{ Type of resection } \\
\hline Pancreatoduodenectomy & $2283(99)$ & $33(32)$ & $8(4)$ & \multirow[t]{3}{*}{$<.0001$} \\
\hline Distal pancreatectomy & $0(0)$ & $64(61)$ & $196(95)$ & \\
\hline Other/unknown & $28(1)$ & $7(7)$ & $2(1)$ & \\
\hline \multicolumn{5}{|l|}{ Margin status } \\
\hline RO & $1428(62)$ & $60(58)$ & $131(63)$ & \multirow[t]{3}{*}{.066} \\
\hline R1-2 & $712(31)$ & $32(31)$ & $51(25)$ & \\
\hline Unknown & $171(7)$ & $12(11)$ & $24(12)$ & \\
\hline \multicolumn{5}{|l|}{$\geq 10$ examined lymph nodes } \\
\hline Yes & $1320(57)$ & $57(55)$ & $95(46)$ & \multirow[t]{3}{*}{.016} \\
\hline No & $922(40)$ & $46(44)$ & $106(52)$ & \\
\hline Unknown & $69(3)$ & $1(1)$ & $5(2)$ & \\
\hline \multicolumn{5}{|c|}{ Number of positive lymph nodes } \\
\hline 0 & $680(29)$ & $43(41)$ & $91(44)$ & \multirow[t]{4}{*}{$<.0001$} \\
\hline $1-3$ & 966 (42) & $38(37)$ & $85(41)$ & \\
\hline$\geq 4$ & $609(26)$ & $16(15)$ & $20(10)$ & \\
\hline Ūnknown & $56(3)$ & $7(7)$ & $10(5)$ & \\
\hline \multicolumn{5}{|c|}{ Death $\leq 30$-day postoperatively } \\
\hline Yes & $83(4)$ & $6(6)$ & $7(3)$ & \multirow[t]{2}{*}{.501} \\
\hline No & $2228(96)$ & $98(94)$ & $199(97)$ & \\
\hline \multicolumn{5}{|c|}{ Death $\leq 90$-day postoperatively } \\
\hline Yes & $154(7)$ & $11(11)$ & $12(6)$ & \multirow[t]{2}{*}{.256} \\
\hline No & 2157 (93) & $93(89)$ & $194(94)$ & \\
\hline \multicolumn{5}{|l|}{ Preoperative chemotherapy ${ }^{a}$} \\
\hline Yes & $67(3)$ & $11(11)$ & $5(2)$ & \multirow[t]{2}{*}{$<.0001$} \\
\hline No & $2244(97)$ & $93(89)$ & $201(98)$ & \\
\hline \multicolumn{5}{|l|}{ Adjuvant chemotherapy } \\
\hline Yes & $1038(45)$ & $42(40)$ & $93(45)$ & \multirow[t]{2}{*}{657} \\
\hline No & $1273(55)$ & $62(60)$ & $113(55)$ & \\
\hline
\end{tabular}

${ }^{a}$ Neoadjuvant or induction chemotherapy before tumor resection.

${ }^{*} p$-value indicates significance of the Chi-square test or Fisher's Exact test as appropriate. proportion treated with chemotherapy was $15 \%, 30 \%$ and $20 \%$ for the patients with a PDAC in the head, body or tail, respectively. In unadjusted analyses, the odds of receiving chemotherapy were higher for both tumor locations body and tail compared to tumor location head. However, after adjustment for patient and tumor characteristics, only patients with a PDAC in the body were more likely to receive chemotherapy compared to patients with a PDAC in the head (adjusted OR 2.03, 95\% Cl 1.64-2.51) (Table 2). Median overall survival in this subgroup was 5.2 months for head, 6.1 months for body and 4.6 months for tail (Figure 2(C)). In unadjusted analysis, survival was better for patients with a PDAC in the body than for patients with a PDAC in the head. However, in multivariable analysis, survival was similar (Table 4).

\section{Patients with distant metastases}

There were 9,952 patients with distant metastases included in the study. The proportions of patients treated with chemotherapy by tumor location were: $22 \%$ for head, $30 \%$ for body and $28 \%$ for tail. Both in univariable and multivariable analyses, the odds of receiving chemotherapy was higher for patients with a PDAC in the body (adjusted OR $1.46,95 \% \mathrm{Cl} 1.28-1.66)$ and for patients with a PDAC in the tail (adjusted OR 1.24, 95\% Cl 1.11-1.39) compared to patients with a PDAC in the head (Table 2).

Median overall survival was 2.6 months for PDAC in the head, 2.4 months for PDAC in the body and 1.9 months for PDAC in the tail (Figure 2(D)). Survival was worse for patients with a PDAC in the body and tail compared to patients with a PDAC in the head (body: adjusted HR $1.17,95 \% \mathrm{Cl}$ 1.10-1.23; and tail: adjusted HR 1.35, 95\% Cl 1.29-1.41) (Table 4).

\section{Discussion}

This first population-based analysis focusing on the association between PDAC location and tumor stage, treatment, and survival found that PDAC locations in the pancreatic body or tail are larger, more often metastasized and less

Table 4. Cox regression analyses.

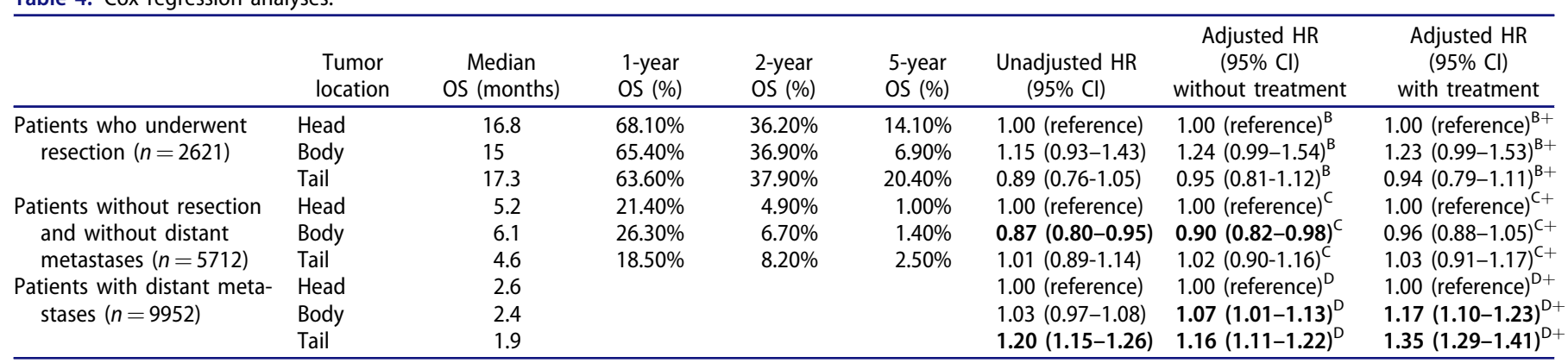

Note: Bold text indicates statistically significant findings.

${ }^{B}$ Odds ratio adjusted for gender, age, period of diagnosis and tumor stage; + (neo)adjuvant chemotherapy.

COdds ratio adjusted for gender, age, period of diagnosis and tumor stage; + palliative chemotherapy.

DOdds ratio adjusted for gender, age and period of diagnosis; + palliative chemotherapy. 
often resectable than PDAC in the pancreatic head. Only in the patients with metastasized disease, overall survival of patients with a PDAC in the body or tail was significantly worse than of patients with a PDAC in the head.

This is the first study in which tumor, treatment and survival of PDAC in head, body and tail are investigated separately. Our findings are in line with previous studies $[5,7,8,11]$. One study describes similar resection rates for body and tail PDAC compared to head PDAC [11]. In contrast, in our study, patients with a PDAC in the body were less likely to undergo resection and patients with a PDAC in the tail are more likely to undergo resection than patients with a PDAC in the head. Because PDAC locations in the body are in close relation to the celiac trunk and major vascular structures (e.g., superior mesenteric artery, portomesenteric vein), these tumors are more often considered unresectable. In contrast, a non-metastasized PDAC in the tail with ingrowth in nearby, less vital, structures (e.g., stomach, spleen, adrenal gland) could more often undergo resection than a PDAC in the head. In some patients with PDAC in the head, malignant biliary obstruction and complications after preoperative biliary drainage, lead to delay or even omission of surgery $[12,13]$. As this is not the case for patients with a PDAC in the tail, this could also partially explain the higher likelihood of resection for tail versus head.

Patients with PDAC in the body who did not undergo resection and had no distant metastases were more often treated with chemotherapy than patients with a location in the head, which could indicate the primary effort to preoperatively reduce these tumors that are more challenging to resect. This is in line with the higher proportion of patients with a resected PDAC in the body receiving preoperative chemotherapy compared with head or tail. In the Netherlands, neoadjuvant chemo(radio)therapy was mostly used in the context of the PREOPANC trial [14] since 2013 for (borderline) resectable PDAC and in the PELICAN trial (NTR5517) for irresectable PDAC since 2014.

Although palliative chemotherapy is advised in the Dutch guideline for pancreatic cancer [15], the proportion of patients with metastases receiving chemotherapy was low in this study (22-30\%). Previously reported rates of palliative chemotherapy are inconsistent and range from $17 \%$ to $54 \%$ [16-20]. If patients are fit, palliative chemotherapy should be considered as our data in accordance with previous literature show this can improve survival significantly $[21,22]$.

Overall, the initiation rate of the adjuvant chemotherapy was low during our study period (48-51\%). While this treatment strategy is widely accepted as standard of care, low initiations rates have also been described in the literature, ranging from $51 \%$ to $68 \%$ in various studies [23-26]. This finding may be attributed to several factors. First, in the Netherlands, adjuvant chemotherapy rates ranged from $26 \%$ to $74 \%$ between 19 pancreatic surgery centers [27]. We expect this difference to be even larger in non-expert/referral centers. Perhaps clinicians interpret the guideline differently and therefore advice on adjuvant treatment may differ. Many patients receive adjuvant chemotherapy in smaller referral centers closer to their home. It was previously shown that high center volume for metastatic pancreatic cancer was associated with more receipt of palliative chemotherapy [16], and this association could also exist for adjuvant treatment. Second, pancreatic surgery has a high complication rate. It is known that a substantial proportion of patients recover too late for the start of adjuvant therapy. Third, patients may choose to not undergo treatment with chemotherapy as they may believe that it will decrease quality of life.

Survival differences between the different tumor locations varied per disease stage. In our study, patients without metastases had similar overall survival for the three locations. This is in contrast with the literature, as better survival is described for head, body and tail PDAC of local/regional disease stage in various studies $[7,8]$. In our study, patients with metastases from head PDAC showed significantly better overall survival than patients with body or tail PDAC, while previous studies found this for metastasized head as well as metastasized tail PDAC $[7,8]$. We found a 5 -year overall survival after pancreatic resection of $20 \%$ for tail, $14 \%$ for head, yet $7 \%$ for body. This is in line with better 3-year relative survival for body/tail cancers as described previously $(20 \%$ body/tail versus $9 \%$ head) [7]. It is unclear from that study whether patients with overlapping locations were included and whether pathological or clinical stage was used for the selection of patients [7]. Besides, both studies included earlier time periods in which other TNM editions were used $[7,8]$. A clear explanation for the lower 5-year overall survival for PDAC in the body was not found. Resection margin and chemotherapy rates were similar to the other tumor locations, and the proportion of patients without positive lymph nodes was even significantly higher than in patients with a PDAC in the head $(41 \%$ versus $29 \%, p<.001)$. Potentially, patients with a PDAC in the body more often have vascular involvement due to the nearby celiac trunk, as compared to other tumor locations, which may lead to worse long-term outcomes. Considering the outcome variations between body and tail, the two locations should be assessed separately in future studies.

It is known that PDAC in the body or tail are often diagnosed later due to the lack of specific clinical symptoms, which leads to detection bias and possibly explains survival differences according to the tumor location. On the other hand, Dreyer et al. recently showed that PDAC in the body and tail may have more aggressive tumor biology compared to PDAC in the head which may explain worse clinical outcomes [10]. Within the group with distant metastases, both a delayed diagnosis and aggressive tumor biology could contribute to the survival difference.

There are several limitations to this study. Firstly, in the Netherlands Cancer Registry, no comorbidity, postoperative morbidity data and cause of death are available. In addition, type of chemotherapy was only registered since 2015 and could therefore not be analyzed in this study. Secondly, a recent study suggested that the Netherlands Cancer Registry underestimates pancreatic cancer incidence of elderly patients, leading to a small overestimation of survival in this study [28]. Thirdly, patients with registered tumor location 'other' were excluded (14\%). In this group, tumor locations 
were partly overlapping (e.g., head/body or body/tail) or 'not otherwise specified' and could therefore not be assigned to the groups of this study. It is possible that some of the overlapping tumors are larger than tumors confined to either head, body or tail, possibly with worse outcomes. As a result, survival in this study could be slightly overestimated.

However, the strength of these Netherlands Cancer Registry data is that it is population based. A large nationwide cohort of patients with PDAC in the Netherlands represents daily clinical practice.

In conclusion, PDAC locations in the pancreatic body or tail are larger, more often metastasized and less often resectable than PDAC in the pancreatic head. Only in patients with metastatic disease, overall survival of patients with a PDAC in the body or tail was significantly worse than of patients with a PDAC in the head.

\section{Ethics approval and consent to participate}

According to the Central Committee on Research involving Human Subjects (CCMO), this type of study does not require approval from an ethics committee in the Netherlands. This study was approved by the Privacy Review Board of the Netherlands Cancer Registry. The study was performed in accordance with the Declaration of Helsinki.

\section{Acknowledgements}

The authors thank the registration team of the Netherlands Cancer Registry for their dedicated data collection and Pauline Vissers (Netherlands Comprehensive Cancer Organization (IKNL)) for performing several analyses.

\section{Disclosure statement}

No potential conflict of interest was reported by the authors.

\section{Funding}

The Dutch Pancreatic Cancer Project, including the Netherlands Cancer Registry, received funding from the Dutch Cancer Society KWF Kankerbestrijding; [grant no. UVA2013-5842].

\section{ORCID}

Tara M. Mackay (D) http://orcid.org/0000-0002-3730-6893

\section{References}

[1] Carrato A, Falcone A, Ducreux M, et al. A systematic review of the burden of pancreatic cancer in Europe: real-world Impact on survival, quality of life and costs. J Gastrointest Canc. 2015;46: 201-211.

[2] Lepage C, Capocaccia R, Hackl M, et al. Survival in patients with primary liver cancer, gallbladder and extrahepatic biliary tract cancer and pancreatic cancer in Europe 1999-2007: results of EUROCARE-5. Eur J Cancer. 2015;51:2169-2178.
[3] van der Geest LG, van Rijssen LB, Molenaar IQ, et al. Volume-outcome relationships in pancreatoduodenectomy for cancer. HPB. 2016;18:317-324.

[4] van der Geest LG, Besselink MG, van Gestel YR, et al. Pancreatic cancer surgery in elderly patients: Balancing between short-term harm and long-term benefit. A population-based study in the Netherlands. Acta Oncologica. 2016;55:278-285.

[5] Ruess DA, Makowiec F, Chikhladze S, et al. The prognostic influence of intrapancreatic tumor location on survival after resection of pancreatic ductal adenocarcinoma. BMC Surg. 2015;15:123.

[6] Neoptolemos JP, Palmer DH, Ghaneh P, et al. Comparison of adjuvant gemcitabine and capecitabine with gemcitabine monotherapy in patients with resected pancreatic cancer (ESPAC-4): a multicentre, open-label, randomised, phase 3 trial. Lancet. 2017; 389:1011-1024.

[7] Lau MK, Davila JA, Shaib YH. Incidence and survival of pancreatic head and body and tail cancers: a population-based study in the United States. Pancreas. 2010;39:458-462.

[8] Sener SF, Fremgen A, Menck HR, et al. Pancreatic cancer: a report of treatment and survival trends for 100,313 patients diagnosed from 1985-1995, using the National Cancer Database. J Am Coll Surg. 1999;189:1-7.

[9] Toomey P, Hernandez J, Golkar F, et al. Pancreatic adenocarcinoma: complete tumor extirpation improves survival benefit despite larger tumors for patients who undergo distal pancreatectomy and splenectomy. J Gastrointest Surg. 2012;16: 376-381.

[10] Dreyer SB, Jamieson NB, Upstill-Goddard R, et al. Defining the molecular pathology of pancreatic body and tail adenocarcinoma. Br J Surg. 2018;105:e183-e191.

[11] Brennan MF, Moccia RD, Klimstra D. Management of adenocarcinoma of the body and tail of the pancreas. Ann Surg. 1996;223: 506-511. discussion 511-502.

[12] Dumonceau JM, Tringali A, Blero D, et al. Biliary stenting: indications, choice of stents and results: European Society of Gastrointestinal Endoscopy (ESGE) clinical guideline. Endoscopy. 2012;44:277-298.

[13] van der Gaag NA, Rauws EA, van Eijck CH, et al. Preoperative biliary drainage for cancer of the head of the pancreas. N Engl J Med. 2010;362:129-137.

[14] Versteijne E, van Eijck $\mathrm{CH}$, Punt $\mathrm{CJ}$, et al. Preoperative radiochemotherapy versus immediate surgery for resectable and borderline resectable pancreatic cancer (PREOPANC trial): study protocol for a multicentre randomized controlled trial. Trials. 2016;17:127.

[15] Landelijke werkgroep Gastro-intestinale tumoren. Richtlijn pancreascarcinoom. Versie 2.0. Integraal Kankercentrum Nederland; 2011. [cited 2018 Sep 10]. Available from: http://oncoline.nl/ pancreascarcinoom.

[16] Haj Mohammad N, Bernards N, Besselink MG, et al. Volume matters in the systemic treatment of metastatic pancreatic cancer: a population-based study in the Netherlands. J Cancer Res Clin Oncol. 2016;142:1353-1360.

[17] Burmeister EA, O'Connell DL, Beesley VL, et al. Describing patterns of care in pancreatic cancer: a population-based study. Pancreas. 2015;44:1259-1265.

[18] David M, Lepage $C$, Jouve $\mathrm{JL}$, et al. Management and prognosis of pancreatic cancer over a 30-year period. Br J Cancer. 2009;101: 215-218.

[19] Oberstein PE, Hershman DL, Khanna LG, et al. Uptake and patterns of use of gemcitabine for metastatic pancreatic cancer: a population-based study. Cancer Invest. 2013;31:316-322.

[20] Sharp L, Carsin AE, Cronin-Fenton DP, et al. Is there under-treatment of pancreatic cancer? Evidence from a population-based study in Ireland. Eur J Cancer. 2009;45:1450-1459.

[21] Burris HA, 3rd, Moore MJ, Andersen J, et al. Improvements in survival and clinical benefit with gemcitabine as first-line therapy for patients with advanced pancreas cancer: a randomized trial. J Clin Oncol. 1997;15:2403-2413. 
[22] Conroy T, Desseigne F, Ychou M, et al. FOLFIRINOX versus gemcitabine for metastatic pancreatic cancer. N Engl J Med. 2011;364: 1817-1825.

[23] Mayo SC, Gilson MM, Herman JM, et al. Management of patients with pancreatic adenocarcinoma: national trends in patient selection, operative management, and use of adjuvant therapy. J Am Coll Surg. 2012;214:33-45.

[24] Merkow RP, Bilimoria KY, Tomlinson JS, et al. Postoperative complications reduce adjuvant chemotherapy use in resectable pancreatic cancer. Ann Surg. 2014;260:372-377.

[25] Sata N, Kurashina K, Nagai H, et al. The effect of adjuvant and neoadjuvant chemo(radio)therapy on survival in 1,679 resected pancreatic carcinoma cases in Japan: report of the national survey in the 34th annual meeting of Japanese Society of Pancreatic Surgery. J Hepatobiliary Pancreat Surg. 2009;16: 485-492.

[26] Watanabe I, Sasaki S, Konishi M, et al. Onset symptoms and tumor locations as prognostic factors of pancreatic cancer. Pancreas. 2004;28:160-165.

[27] Bakens MJ, van der Geest LG, van Putten M, et al. The use of adjuvant chemotherapy for pancreatic cancer varies widely between hospitals: a nationwide population-based analysis. Cancer Med. 2016;5:2825-2831.

[28] Fest J, Ruiter R, van Rooij FJ, et al. Underestimation of pancreatic cancer in the national cancer registry - Reconsidering the incidence and survival rates. Eur J Cancer. 2017;72:186-191. 